\title{
Manía e hipomanía inducida por antidepresivos en niños y adolescentes. Revisión bibliográfica
}

\author{
Mania and hypomania induced by antidepressants in children and adolescents. Bibliogra- \\ phic review
}

\section{Estefanía Adela León'}

\section{Resumen}

Los niños son más propensos a los efectos adversos de los inhibidores selectivos de la recaptación de serotonina que los adultos. El objetivo del presente trabajo es revisar la bibliografía sobre la manía y la hipomanía inducida por estos antidepresivos. Se encontró que gran parte de los jóvenes con diagnóstico de trastorno bipolar habían estado expuestos a este tipo de fármacos. Mientras que la (hipo)manía asociada a antidepresivos en niños con trastornos de ansiedad es tan relevante como la de aquellos que previamente habían recibido un diagnóstico de depresión. Como limitación, en los trabajos seleccionados, el síndrome de activación podía incluir o no a la (hipo)manía. Al indicar un antidepresivo debemos ser sumamente prudentes y hacer especial énfasis en el análisis riesgo-beneficio. Es imprescindible realizar una búsqueda exhaustiva de antecedentes familiares de trastorno bipolar junto con una pesquisa de antecedentes personales y un minucioso análisis semiológico de nuestros pacientes debido a los riesgos de (hipo)manía.

Palabras clave: Hipomanía - Manía - Inhibidores Selectivos de la Recaptación de Serotonina - Niños - Adolescentes.

\begin{abstract}
Children are more prone to adverse effects of selective serotonin reuptake inhibitors than adults. The aim of this study is to review the bibliography on mania and hypomania induced by these antidepressants. Most of the young people diagnosed with bipolar disorder had been exposed to this type of drug. The hypomania associated with these antidepressants in children with anxiety disorders is as relevant as that of those who had previously received a diagnosis of depression. As a limitation, in the selected papers, the activation syndrome could or could not include (hypo) mania. When indicating an antidepressant, we must be extremely prudent and place special emphasis on the risk-benefit analysis. It is essential to conduct a thorough search of family history of bipolar disorder together with a personal history investigation and a meticulous analysis of the semiology of our patients due to the risks of (hypo) mania.
\end{abstract}

Keywords: Hypomania - Mania - Selective Serotonin Reuptake Inhibitors - Children - Adolescents.

RECIBIDO 2I/I/202I - ACEPTADO 10/4/202I

'. Médica especialista en psiquiatría infanto-juvenil. Magister en ciencias, tecnologías y salud, mención biología integrativa especializada en neurociencias (M2). Sorbonne Université, París, Francia. Psiquiatra infanto juvenil del Hospital Interzonal de Agudos Especializado en Pediatría "Sor María Ludovica" y del Centro de Salud n³ "Dr.Arturo Ameghino".

Correspondencia:

17estefanialeon@gmail.com

Lugar de realización del estudio: H.I.A.E.P.“Sor María Ludovica”, La Plata, Buenos Aires. 


\section{Introducción}

Los Inhibidores Selectivos de la Recaptación de Serotonina (ISRS) son prescriptos asociados a psicoterapia para el tratamiento de trastornos del humor, trastornos de ansiedad (TA) y trastornos obsesivo compulsivos (TOC) en niños, adolescentes y adultos. Como ventaja por sobre los antidepresivos tricíclicos (ADT) son altamente selectivos para el transportador 5-HT por lo que presentan un mejor perfil de seguridad y tolerabilidad.

Sin embargo, no es posible dar por supuesto que los efectos adversos en niños y adolescentes sean iguales a los que presentan los adultos debido a diferencias farmacocinéticas y farmacodinámicas. Como en el caso de los ADT que son efectivos en la depresión del adulto pero según una revisión Cochrane (Hazell et al., 2013) no son útiles en el tratamiento de la depresión en niños prepúberes (Gordon \& Melvin, 2013; Hazell et al., 2013).

La prevalencia de utilización de antidepresivos (AD) en pediatría ha variado considerablemente desde la Black-Box Warning emitida por la FDA en 2004. Dicha entidad advirtió sobre un aumento del riesgo de pensamientos, sentimientos y comportamientos suicidas en los jóvenes que consumen $\mathrm{AD}$.

No obstante, los ensayos incluidos en el metaanálisis por el cual se emitió la advertencia no fueron diseñados para estimar riesgo suicida. En los EE. UU. se observó una abrupta disminución del uso de $\mathrm{AD}$ en el $2^{\circ}$ año posterior a las advertencias (31\%, IC 95\%). Pero, se halló un importante aumento de intoxicaciones por drogas psicotrópicas (del 10.0\% a 17.8\%) (Bachmann et al., 2016).

Bridge J. et al. (2007) concluyeron en su metaanálisis que los beneficios de los ISRS parecen ser mucho mayores que los riesgos de ideación suicida e intento de suicidio en todas las indicaciones.

Empero, la evidencia indica una pequeña superioridad de los ISRS por sobre el placebo en la depresión pediátrica (Locher et al., 2017).

Bachmann C. et al. (2016) evaluaron la prescripción de $\mathrm{AD}$ de 2005 a 2012. Encontraron que su uso se incrementó de $1.3 \%$ a $1.6 \%$ en Estados Unidos (+ $26.1 \%$ ) y de $0.7 \%$ a $1.1 \%$ en Reino Unido (+ $54.4 \%)$. Siendo los ISRS los AD más recetados. (3)

Al momento actual, los ISRS aprobados en pediatría por la FDA son sertralina ( $\geq 6$ años: TOC), fluoxetina ( $\geq 7$ años: TOC; $\geq 8$ años: TDM $y \geq 10$ : en combinación con olanzapina para depresión bipolar), escitalopram ( $\geq 12$ años: TDM); fluvoxamina ( $\geq 8$ años: TOC), duloxetina ( $\geq 7$ años; trastorno de ansiedad generalizada).
También se prescriben para indicaciones off label como las conductas repetitivas en niños con trastornos del espectro autista (Carrasco et al., 2012).

Sus efectos adversos son físicos o psiquiátricos. Estos últimos pueden ser divididos de distintas maneras. Por un lado, efectos adversos del espectro de manía (manía, hipomanía), del espectro de depresión (agravación de la depresión, llanto, irritabilidad, ira, hipersensibilidad), del espectro de agitación (agitación, acatisia, inquietud, nerviosismo), síntomas de ansiedad, pánico y temblor.

Por el otro, el síndrome de activación que suele incluir irritabilidad, agitación, manifestaciones somáticas de la ansiedad, ataques de pánico, inquietud, agresión e insomnio. Pero puede o no incluir acatisia, desinhibición, hipomanía y manía. Según Luft M. et al. (2018) carece de una definición clara aun cuando se lo considera un factor de riesgo de interrupción de la medicación y algunos autores lo asocian a suicidio.

Los niños son más propensos a los efectos adversos psiquiátricos de los ISRS que los adultos. Sin embargo, la mayoría de sus estudios en población pediátrica están diseñados para evaluar eficacia y no efectos adversos (Amitai et al., 2015; Gordon \&Melvin, 2018; Luft et al., 2018; Naguy, 2016).

Los síntomas hipomaníacos pueden propiciar un deterioro psicosocial significativo y estar asociados con un mayor riesgo de suicidio, abuso de sustancias, problemas académicos y legales.

Además, la edad más temprana del primer tratamiento $\mathrm{AD}$ sería un predictor de vulnerabilidad a la aceleración del ciclo inducida por AD (Birmaher, 2013).

Por lo anteriormente mencionado el objetivo del presente trabajo es revisar la bibliografía disponible sobre la manía y la hipomanía inducida por el tratamiento (MHIT) con ISRS en niños y adolescentes.

\section{Metodología}

Revisión bibliográfica selectiva. Las bases de datos utilizadas fueron: PubMed y Biblioteca Cochrane.

\section{Resultados}

Según Faedda G. et al. (2004) cuando un niño o un adolescente presentan un cuadro de manía asociada al uso de AD lo acontecido suele ser inesperado y dramáticamente disruptivo. No obstante, debemos hacer énfasis en la búsqueda de síntomas hipomaníacos que deben exceder las expectativas para la etapa de desarrollo normal (Birmaher, 2013; Faeddaa, 2004). 


\section{Breve reseña en adultos}

De los primeros 500 participantes del estudio STEP$\mathrm{BD}$ con $\mathrm{TB}, 57,6 \%$ (n 288) tenían antecedentes de tratamiento AD. De estos, el $44 \%$ reportaron al menos un cambio anímico (manía/hipomanía/episodio mixto) dentro de las primeras 12 semanas del inicio del AD. Los autores concluyeron que la MHIT es más frecuente en individuos bipolares con corta duración de la enfermedad y en aquellos con múltiples ensayos anteriores con AD (Truman et al., 2007).

Henry C. et al. (2001) encontraron que el $27 \%$ de los 44 pacientes con TB que incluyeron presentaron MHIT. Las mismas fueron menores al asociar el AD a litio y mayores en personas con temperamento hipertímico (Henry et al., 2001).

La quinta versión del Manual Diagnóstico y Estadístico de los Trastornos Mentales (DSM-5) menciona, por primera vez, que los individuos sin antecedentes de TB que presenten MHIT en intensidad y duración suficientes pueden ser considerados bipolares. Empero, no tiene criterios específicos para el diagnóstico de TB pediátrico (TBP) (de Dios et al., 2014).

Por otra parte, la clasificación de TB de Akiskal define como TB III a las depresiones con hipomanía inducida por fármacos (Akiskal et al., 2003) y propone como hipótesis causales: a) TB no diagnosticado; b) inducción de novo de TB y c) efectos adversos farmacológicos. (Akiskal et al., 2003; Luft et al., 2018).

\section{Prescripción de ISRS en el TBP}

De los 245 pacientes con TBP que incluyeron Pagano $\mathrm{M}$ et. al. (2008), el 46\% tenían antecedentes de haber recibido AD (64\% ISRS). Siendo la edad promedio del primer uso de 9.52 años ( $\mathrm{SD}=3.26$ años), con una duración promedio del tratamiento cercana a un año [media 0.86 años (DS 1.31)] (Akiskal et al., 2003).

Particularmente los hijos de padres bipolares están frecuentemente expuestos a psicofármacos. Chang K. et. al (2010) incluyeron 43 hijos con bipolaridad subsindromal con una edad media de $12.3 \pm 2.8$ años; $42 \%$ tenía al menos dos diagnósticos psiquiátricos comórbidos (el 100\% de Trastorno por déficit de atención e hiperactividad-TDAH-). A su vez, el 39,5\% habían recibido ISRS. Siendo el promedio de medicamentos psicotrópicos que alguna vez tomaron de $2.7 \pm 1.9$ (Offidani et al., 2013).

\section{Ansiedad}

En la revisión sistemática de Offidani E. et al. (2013) realizada en jóvenes las tasas de activación durante el tratamiento $\mathrm{AD}$ fueron relevantes tanto para el TDM
$(11,2 \%)$ como para los TA $(13,8 \%)$, y entre 3 a 10 veces superior al placebo. La frecuencia de MHIT fue considerablemente mayor en los jóvenes con tratamiento $\mathrm{AD}(7,98 \%)$ que en los que se encontraban recibiendo placebo $(0,17 \%)$.

Como hipótesis causales podemos mencionar específicamente la mayor reactividad al entorno social y los TA como vía para el desarrollo de TBP.

Sala R. et al. (2010) realizó un estudio en el cual incluyó 446 participantes con TBP. El 44\% presentó al menos un TA y en la mayoría de los casos precedieron el inicio del TB (78,9\%).

\section{Depresión}

La revisión sistemática de Offidani et al. (2013) encontró que las tasas medias brutas de MHIT fueron 20,5 veces más altas con $\mathrm{AD}(16,8 \pm 14,6 \%)$ que con placebo $(0,82 \pm 0,86 \%)$ en adolescentes con TDM.

En la revisión de Cheung et al. (2005), la tasa media de manía fue del $2 \%$, la mayor incidencia con fluoxetina (6\%); para el placebo, la incidencia fue de 0 a $2 \%$. En la mayoría de los casos los síntomas se resolvieron al disminuir la dosis o suspender el AD (21).

Geller et al. (2001) reportaron que los individuos que tenían TDM prepúberal tuvieron tasas significativamente más altas en la adultez (edad media 20,7 años, $\mathrm{SD}=2,0)$ de $\mathrm{TB}$ que los sujetos normotipicos (48.6\% [ $\mathrm{N}=35]$ versus $7.1 \%[\mathrm{~N}=2])$.

Faedda G. et al. (2004) observó que de 82 pacientes con TBP el 69\% habían recibido $\mathrm{AD}$ al menos una vez en su vida y de estos el 58\% presentaron MHIT.

\section{Diagnóstico de Trastorno Depresivo Bi- polar (TDB)}

Según Birmaher (2014) el TDB es un trastorno recurrente con síndromes frecuentes y especialmente episodios depresivos y subsindrómicos mixtos que generan un deterioro significativo del funcionamiento psicosocial.

Su diagnóstico resulta particularmente difícil debido a la inmadurez propia de los niños, la mayor frecuencia de inicio depresivo, la presencia de trastornos comórbidos y las interpretaciones divergentes de la sintomatología maníaca por parte de los profesionales. Los jóvenes con manía subsindrómica y antecedentes familiares de primer grado TB tienen alto riesgo de desarrollar TB (Birmaher, 2013).

Nassir Ghaemi (2004) propuso criterios para diferenciar la depresión bipolar de la unipolar en adultos. La primera presentación del TB suele ser depresiva y por definición el TBP cumple con el criterio D6 pro- 
puesto de inicio en menores de 25 años. A su vez, los adolescentes deprimidos parecen tener más sintomatología atípica (D4), como la hipersomnia, el incremento del apetito y aumento de peso en comparación con los adultos. Reportes indican que hasta el $40 \%$ de los jóvenes con TBP pueden tener síntomas psicóticos (D5). Es de suma trascendencia indagar la presencia de antecedentes familiares de TB y depresión posparto (Geller et al., 2001).

El TDB puede aparecer en cualquier momento del tratamiento, sin embargo estudios reportan que sería más probable al inicio del mismo (2-3 semanas).

La mayoría de los autores sugieren que dosis más altas se asocian a mayor frecuencia de síndrome de activación y la disminución de la dosis podría tener efectos beneficiosos.

\section{Influencia de la edad}

El síndrome de activación con ISRS es más frecuente en niños que en adolescentes.

En niños varió de $8 \%$ a $17 \%$ con un promedio de $10.7 \%$ para ISRS y de 3,4\% para el placebo. En los adolescentes las tasas de activación promedio fueron $2.1 \%$ para ISRS y 1,9\% para placebo (Amitai et al., 2015).

\section{Discusión y conclusiones}

Tal como se hace mención en la revisión realizada por Goldsmith et. al (2011) es una preocupación para los psiquiatras infanto juveniles la posibilidad de que la exposición a antidepresivos pueda acelerar o causar un inicio temprano de TB. En la práctica clínica encontramos niños y adolescentes que presentan un cuadro de MHIT. Por un lado, con TA y sin antecedentes de TB personales ni familiares, ni de TDAH que responden a: a) la disminución de la dosis del $\mathrm{AD}$ o b) la suspensión del psicofármaco o c) en el caso de que el trastorno de base sea muy disruptivo, y no se pueda indicar otro medicamento, la adición de antipsicóticos en dosis bajas.

Por el otro, pacientes con diagnóstico de TDM, antecedentes familiares de TB y/o personales de TDAH y/o marcada irritabilidad en los cuales se desencadena cuadros (hipo)maniacos/mixtos que no revierte al suspender el $\mathrm{AD}$, debiéndose iniciar tratamiento para TB. (Amitai et al., 2015; Goldsmith et al., 2011).

Gran parte de los jóvenes con diagnóstico de TB habían estado expuestos a AD. La proporción de niños y adolescentes con TA que presentan MHIT es tan relevante como la de aquellos que previamente habían recibido un diagnóstico de depresión (Faeddaa et al., 2004; Offidani et al., 2013; Pagano et al., 2008).
Como principal limitación en esta revisión en los trabajos seleccionados el síndrome de activación podía incluir o no a la manía y a la hipomanía. A su vez, no se desglosa su frecuencia en particular. Incluso, en algunos estudios los investigadores no describieron como se definieron los síntomas maniacos.

Además, la mayoría de los estudios son retrospectivos. Resulta necesaria la realización de investigaciones prospectivas diseñadas específicamente para la búsqueda de efectos adversos psiquiátricos. A su vez, todavía existen profesionales que consideran los cuadros de hipomanía como "características normales" de los niños lo que podría ser causante de que las prevalencias estén subestimadas.

Para concluir, al indicar un ISRS debemos ser sumamente prudentes y hacer especial énfasis en el análisis riesgo-beneficio. Es imprescindible realizar una búsqueda exhaustiva de antecedentes familiares de TB junto con una pesquisa de antecedentes personales y un minucioso análisis semiológico de nuestros pacientes debido a los riesgos de MHIT. Así mismo, posterior a la prescripción, la monitorización debe ser muy estrecha.

Conflictos de intereses: la autora declara no tener conflictos de intereses. Este artículo tiene como antecedente el trabajo final del curso "Abordaje interdisciplinario del trastorno bipolar", FUBIPA, 2019.

\section{Referencias bibliográficas}

Akiskal, H., Hantouche, E. \& Allilaire, J. (2003). Validating antidepressant-associated hypomania (bipolar III): a systematic comparison with spontaneous hypomania (bipolar II). J Affect Disord, 73: 65-74. doi: 10.1016/ s0165-0327(02)00325-7

Amitai, M., Chen, A., Weizman, A. \& Apter, A. (2015). SSRI-Induced Activation Syndrome in Children and Adolescents-What Is Next? Curr Treat Options Psych, 2(1): 28-37. https://doi.org/10.1007/s40501-015-0034-9

Bachmanna, C., Aagaardb, L., Burcuc, M., Glaeske, G., Kalverdijk, L., Petersen, I., Schuiling-Veninga, C. C. M., Wijlaars L., Zito J. M. , Falk Hoffmann, F. (2016). Trends and patterns of antidepressant use in children and adolescents from five western countries, 2005-2012. Neuropsychopharmacol, 26 (3): 411-4199. doi: 10.1016/j.euroneuro.2016.02.001. Epub 2016 Feb 8.

Birmaher, B. (2013). Bipolar disorder in children and adolescents. Child Adolesc Ment Health, Sep 1; 18(3): 10.1111/camh.12021.

Bridge, J., Iyengar, S., Salary, C., Barbe, R., Birmaher, B., Pincus H. A., Ren, L., Brent D. A. (2007). Clinical Response and Risk for Reported Suicidal Ideation and Suicide Attempts in Pediatric Antidepressant Treatment: A Meta-analysis of Randomized Controlled Trials. JAMA, 297(15):16831696. doi: 10.1001/jama.297.15.1683.

Carrasco, M., Volkmar, F. \& Bloch, M. (2012). Pharmacologic treatment of repetitive behaviors in autism spectrum disorders: evidence of publication bias. Pediatrics, 129(5):e1301-1310. doi: 10.1542/peds.2011-3285.

Chang, K., Saxena, K., Howe, M. \& Simeonova, D. (2010). Psychotropic Medication Exposure and Age at Onset of Bipolar Disorder in Offspring of Parents with Bipolar Disorder. J Child Adolesc Psychopharmacol, 20(1): 25-32. doi: 10.1089/cap.2009.0036. 
de Dios, C., Goikolea, J. Colomm F., Moreno, C., \& Vieta, E. (2014). Los trastornos bipolares en las nuevas clasificaciones: DSM-5 y CIE-11. Rev Psiquiatr Salud Ment, 7(4):179-185. doi: 10.1016/j.rpsm.2014.07.00

Faeddaa, G., Baldessarini, R., Glovinskya, I. \& Austin, N. (2004). Treatment-emergent mania in pediatric bipolar disorder: a retrospective case review. J Affect Disord, 82: 149-158. doi: 10.1016/j.jad.2003.12.011

Geller, B., Zimerman, B., Williams, M., Bolhofer, K. \& Craney, J. (2001). Bipolar Disorder at Prospective Follow-Up of Adults Who Had Prepubertal Major Depressive Disorder. Am J Psychiatry, 158(1): 125-128. doi: 10.1176/appi.ajp.158.1.125.

Ghaemi, N., Hsu, D., Ko, J., Baldassano, C., Kontos, N., Goodwin, F.G. et. al. (2004). Bipolar Spectrum Disorder: A Pilot Study. Psychopathology, 37(5): 222-225. doi: 10.1159/000080717

Goldsmith, M., Singh, M. \& Chang, K. (2011). Antidepressants and psychostimulants in pediatric populations: is there an association with manía? Paediatr Drugs, 13(4): 225-243. doi: 10.2165/11591660-000000000-00000

Gordon, M., Melvin, G. (2013) Selective serotonin re-uptake inhibitors A review of the side effects in adolescents. Aust Fam Physician, (9): 620-623.

Hazell, P., Mirzaie, M. (2013). Tricyclic drugs for depression in children and adolescents. Cochrane Database of Systematic Reviews. https://www. cochranelibrary.com/cdsr/doi/10.1002/14651858.CD002317.pub2/full

Henry, C., Sorbara, F., Lacoste, J., Gindre, C. \& Leboyer, M. (2001). Antidepressant-Induced Mania in Bipolar Patients: Identification of Risk Factors. J Clin Psychiatry, 62(4): 249-255. doi: 10.4088/jcp.v62n0406.

Locher, C., Koechlin, H., Zion, S., Werner, C., Pine, D., Kessler, R.C. \& Kossowsky, J. (2017). Efficacy and Safety of Selective Serotonin Reuptake Inhibitors, Serotonin-Norepinephrine Reuptake Inhibitors, and Placebo for Common Psychiatric Disorders Among Children and Adolescents: A Systematic Review and Meta-analysis. JAMA Psychiatry, 74(10): 10111020. doi: 10.1001/jamapsychiatry.2017.2432.
Luft, M., Lamy, M., DelBello, M., McNamara, R. \& Strawn, J. (2018). Antidepressant-Induced Activation in Children and Adolescents: Risk, Recognition and Management. Curr Probl Pediatr Adolesc Health Care, 48(2): 50-62. doi: 10.1016/j.cppeds.2017.12.001.

Naguy, A. (2016). SSRIs-Related Behavioural Syndromes in Children and Adolescents. J Can Acad Child Teen Psychiatry, 25(2): 69-70. PMCID: PMC4879944

Offidani, E., Fava, G., Tomba, E. \& Baldessarini, R. (2013). Excessive Mood Elevation and Behavioral Activation with Antidepressant Treatment of Juvenile Depressive and Anxiety Disorders: A Systematic Review. Psychother Psychosom, 82(3): 132-141. doi: 10.1159/000345316

Pagano, M., Demeter, C., Faber, J., Calabrese, J. \& Findling, R. (2008). Initiation of stimulant and antidepressant medication and clinical presentation in juvenile bipolar I disorder. Bipolar Disord, 10(2): 334-41. doi: 10.1111/j.13995618.2007.00496.x

Sala, R., Axelson, D., Castro-Fornieles, J., Goldstein, T., Ha, W., Liao, F., Gill M. K., Satish Iyengar, S., Strober, M. A., Goldstein, B. I., Yen, S, Hower, H., Hunt, J., Ryan N. D., Dickstein, D., Keller, M. B. and Birmaher, B. (2010). Comorbid anxiety in children and adolescents with bipolar spectrum disorders: prevalence and clinical correlates. J Clin Psychiatry, 71(10): 1344-1350. doi: 10.4088/JCP.09m05845gre.

Truman, C., Goldberg, J., Ghaemi, N., Baldassano, C., Wisniewski, S, Dennehy, E. B., Thase, M. E. \& Sachs, G. S. (2007). Self-Reported History of Manic/Hypomanic Switch Associated With Antidepressant Use: Data From the Systematic Treatment Enhancement Program for Bipolar Disorder (STEP-BD) [CME]. J Clin Psychiatry, 68(10):1472-1479. doi: 10.4088/ jcp.v68n1002. 